\title{
Capacitively Coupled Silicon-Organic Hybrid Modulator for 200 Gbit/s PAM-4 Signaling
}

\author{
S. Ummethala ${ }^{1,2, *}$, J. N. Kemal ${ }^{1}$, M. Lauermann ${ }^{1,3}$, A. S. Alam ${ }^{1}$, H. Zwickel ${ }^{1}$, T. Harter ${ }^{1}$, Y. Kutuvantavida ${ }^{1}$, \\ L. Hahn' ${ }^{2}$, S. H. Nandam ${ }^{4}$, D. L. Elder ${ }^{5}$, L. R. Dalton ${ }^{5}$, W. Freude ${ }^{1}$, S. Randel ${ }^{1}$, C. Koos ${ }^{1,2, * *}$ \\ ${ }^{1}$ Institute of Photonics and Quantum Electronics (IPQ), Karlsruhe Institute of Technology (KIT), Karlsruhe, Germany \\ ${ }^{2}$ Institute of Microstructure Technology (IMT), Karlsruhe Institute of Technology (KIT), Karlsruhe, Germany \\ ${ }^{3}$ Now with Vanguard Photonics GmbH, Karlsruhe, Germany \\ ${ }^{4}$ Institute of Nanotechonology (INT), Karlsruhe Institute of Technology (KIT), Karlsruhe, Germany \\ ${ }^{5}$ Department of Chemistry, University of Washington, Seattle, WA, USA \\ "sandeep.ummethala@kit.edu, ${ }^{* *}$ christian.koos@kit.edu
}

\begin{abstract}
We demonstrate capacitively coupled silicon-organic hybrid ( $\mathrm{SOH}$ ) modulator with a $\pi$ voltage-length product of $1.3 \mathrm{~V} \mathrm{~mm}$ and $3 \mathrm{~dB}$ EO bandwidth exceeding $65 \mathrm{GHz}$. The modulator is used for 200 Gbit/s (100 GBd) PAM-4 signaling.

OCIS codes: (230.4110) Modulators; (130.3120) Integrated optics devices; (200.4650) Optical interconnects
\end{abstract}

\section{Introduction}

High-speed, low-cost electro-optic modulators with small footprint and low drive voltage are essential for short-reach links within data-center and campus-area networks. In this context, the silicon photonic ( $\mathrm{SiP}$ ) platform is attractive, offering mature high-yield fabrication techniques well suited for highly scalable mass production. Although conventional depletion-type SiP modulators have been pushed to bandwidths of about $50 \mathrm{GHz}$, their efficiency is still rather low, leading to $\pi$-voltage-length products of the order ${ }^{1}$ of $10 \mathrm{~V} \mathrm{~mm}$. Silicon-organic hybrid (SOH) devices can overcome these limitations by combining nanophotonic silicon slot waveguides with highly efficient electro-optic (EO) materials, leading to ultra-low $U_{\pi} L$ products $^{2}$ of $0.32 \mathrm{~V} \mathrm{~mm}$ and symbol rates ${ }^{3}$ of $100 \mathrm{GBd}$. However, the analogue bandwidth of such devices is still limited ${ }^{3}$ to, e.g., $25 \mathrm{GHz}$ by the finite conductivity of the doped silicon slabs that are used to electrically contact the rails of the slot waveguide. Plasmonic-organic hybrid (POH) do not suffer from these limitations and offer bandwidths ${ }^{4}$ of more than $300 \mathrm{GHz}$, but suffer from high optical losses. In an alternative approach, $\mathrm{SiP}$ waveguides can be combined with high- $\kappa$ dielectric materials instead of doped silicon slab ${ }^{5}$. Experimental demonstrations of this concept ${ }^{6}$, however, were still limited to devices with $U_{\pi} L$ products of $14 \mathrm{~V} \mathrm{~mm}$, measured at a frequency of $1 \mathrm{kHz}$.

In this paper, we demonstrate a capacitively coupled $\mathrm{SOH}(\mathrm{CC}-\mathrm{SOH})$ modulator, where the resistive Si slabs of a conventional SOH modulator are replaced by a high- $\kappa$ dielectric, $\mathrm{BaTiO}_{3}(\mathrm{BTO})$. The $\mathrm{BTO}$ layer is used to confine the modulating RF signal to the EO material in the slot, thereby overcoming the bandwidth limitations of conventional resistively-coupled SOH devices. We show that the EO frequency response is extremely broad having a $3 \mathrm{~dB}$ bandwidth of more than $65 \mathrm{GHz}$, and the $\pi$-voltage-length product is as small as $1.3 \mathrm{~V} \mathrm{~mm}$. Using a $1 \mathrm{~mm}$-long device, we generate PAM-4 signals at line rates (symbol rates) of $200 \mathrm{Gbit} / \mathrm{s}(100 \mathrm{GBd})$ with a bit error ratio (BER) of $9 \times 10^{-3}$. To the best of our knowledge, this is the first demonstration of a CC-SOH modulator with competitive performance parameters, leading to the highest PAM-4 data rate so far demonstrated with modulators based on dielectric waveguides on the silicon photonic platform.

\section{Capactively Coupled Silicon-Organic Hybrid (CC-SOH) Modulator}

The CC-SOH Mach-Zehnder modulator (MZM) is fabricated on the silicon-on-insulator (SOI) platform, see Fig. 1(a). Each MZM arm consists of a SiP strip waveguide $(500 \mathrm{~nm} \times 220 \mathrm{~nm})$ which is transformed into a slot waveguide through a logarithmic taper, see Inset of Fig. 1(a). A cross-section of the slot waveguide is shown in Fig. 1(b). It comprises two $200 \mathrm{~nm}$-wide and $220 \mathrm{~nm}$-high silicon rails separated by a $100 \mathrm{~nm}$-wide slot. The RF signal is supplied by a co-planar transmission line made from gold (yellow) in ground-signal-ground (GSG) configuration, see Fig. 1(a). Amorphous BTO slabs (red) having a height of $150 \mathrm{~nm}$, a width of $1 \mu \mathrm{m}$, and a high electric permittivity ${ }^{7}$ of $\varepsilon_{\mathrm{r}}>50$ are deposited between the optical slot waveguide and the transmission line to enhance capacitive coupling of the RF signal to the slot. The finite difference time domain (FDTD) simulations in Fig. 1(b) and (c) show that the optical quasi-TE mode and the RF mode are tightly confined to the slot region and hence have a strong overlap. An organic EO polymer (YLD124) is filled into the silicon slots and is activated by a one-time poling process. To this end, the device is heated and a voltage is applied between the (floating) ground electrodes, allowing the MZM to operate in push-pull mode ${ }^{3}$. A geometrical length difference of $80 \mu \mathrm{m}$ between the MZM arms allows to select the operating point by tuning the wavelength. For a $1 \mathrm{~mm}$-long CC-SOH MZM, we measure a $U_{\pi}$ of $1.3 \mathrm{~V}$.

\section{Bandwidth Measurement of CC-SOH Modulator}

CC-SOH modulators overcome the RC limitations of conventional SOH devices by replacing the resistive slabs with a high-К dielectric material. For short devices, the lumped element equivalent circuit results in a small slot capacitor which is connected to the RF signal lines by a large coupling capacitor rather than a resistor. This coupling is not associated with any time constant, and the bandwidth is only limited by the RF propagation losses of the modulation signal. Fig. 1(d) shows the electro-optic-electric (EOE) response of a $1 \mathrm{~mm}$-long CC-SOH modulator with $150 \mathrm{~nm}$ 

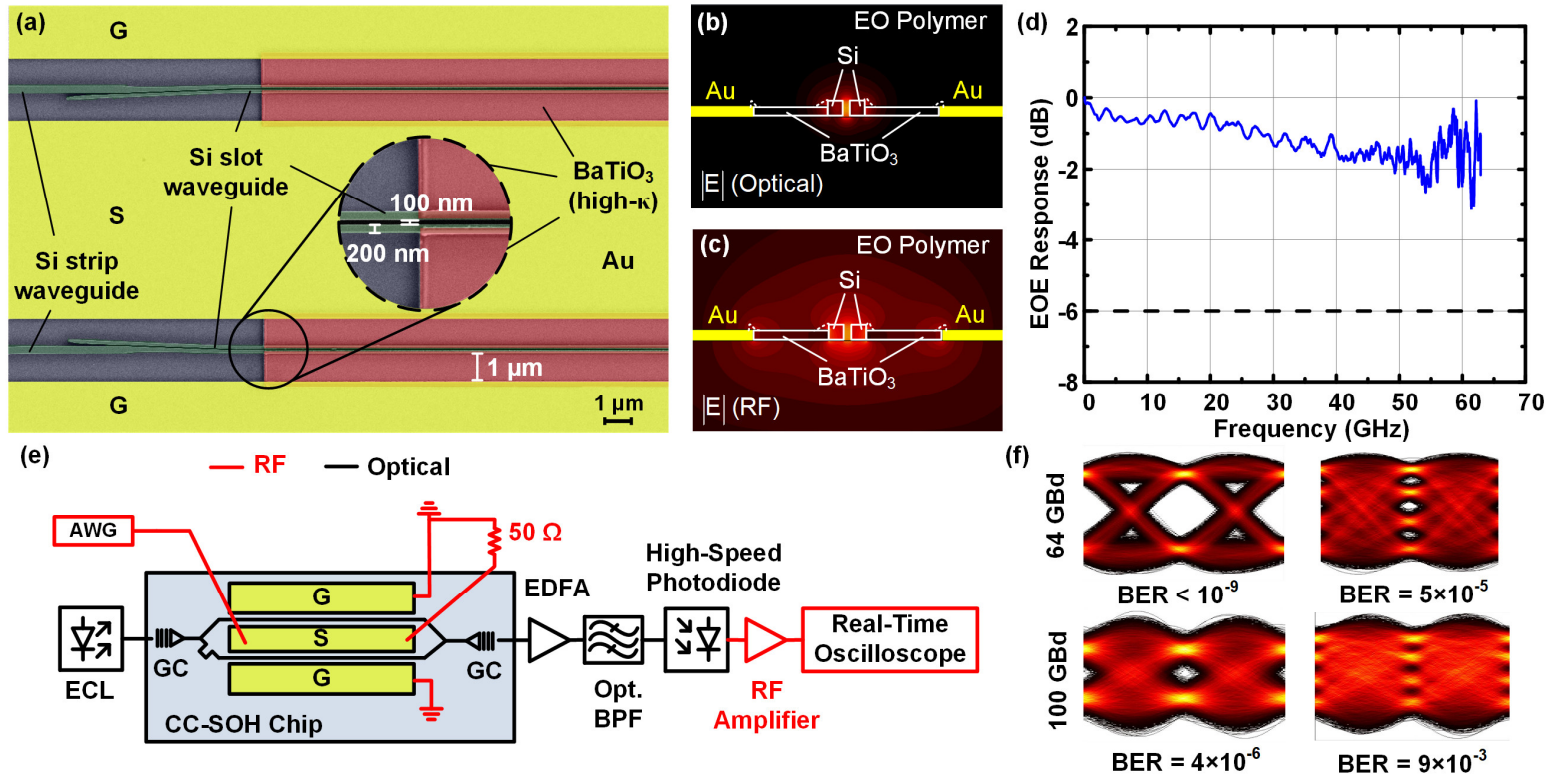

Fig. 1: (a) False-color scanning electron micrograph of CC-SOH MZM. Inset: Silicon slot waveguide surrounded by layers of $\mathrm{BaTiO}_{3}$. (b), (c) Electric field profiles of the optical quasi-TE mode and the RF mode, both tightly confined to the slot region. (d) Measured electro-opticelectric (EOE) response of a $1 \mathrm{~mm}$-long CC-SOH modulator showing a $6 \mathrm{~dB}$ EOE bandwidth well beyond $65 \mathrm{GHz}$. (e) Setup for data transmission experiments. (f) Extracted eye diagrams and bit error ratio (BER) for NRZ and PAM-4 signals at symbol rates of 64 GBd and $100 \mathrm{GBd}$.

thick BTO. The $6 \mathrm{~dB}$ electro-optical-electrical (EOE) bandwidth corresponds to the $3 \mathrm{~dB}$ electro-optic (EO) bandwidth, which is well beyond the $65 \mathrm{GHz}$ that is accessible by the vector network analyzer used in our setup.

\section{Data Transmission Experiments}

To evaluate the transmission performance of the CC-SOH modulator, we use the setup depicted in Fig. 1(e). The electrical signals are generated by a $120 \mathrm{GSa} / \mathrm{s}$ arbitrary-waveform generator (AWG, Keysight M8194A, analog bandwidth $45 \mathrm{GHz}$ ). The resulting signal drives the GSG electrodes of the MZM via microwave probes having a nominal bandwidth of $67 \mathrm{GHz}$. An external $50 \Omega$ impedance terminates the coplanar transmission line. We use sinc-like pulse shapes featuring a raised-cosine spectrum for generating signals with symbol rates of $64 \mathrm{GBd}$ and $100 \mathrm{GBd}$. The peakto-peak on-chip drive voltage is less than $1 \mathrm{~V}$ for $100 \mathrm{GBd}$ signaling. An external-cavity laser (ECL) provides the optical carrier at a wavelength near $1550 \mathrm{~nm}$. Light is coupled in and out of the CC-SOH chip via grating couplers. An erbium-doped fiber amplifier (EDFA) compensates the fiber-to-fiber insertion loss of the device, which amounts to $22 \mathrm{~dB}$. Note that this loss is mainly caused by non-optimum grating couplers $(9 \mathrm{~dB}$ total) and waveguide design, both of which can be further improved. The modulated optical carrier is filtered by a $2 \mathrm{~nm}$ optical band-pass filter and fed to a high-speed photodiode with a bandwidth of $70 \mathrm{GHz}$ (Finisar XPDV3120R). The resulting electrical signal is amplified by an RF amplifier and recorded with an oscilloscope having an analog bandwidth of $100 \mathrm{GHz}$ (Keysight UXR1004A). A programmed pre-compensation in the AWG accounts for the strong frequency roll-off of the AWG and other RF components at the transmitter, excluding the modulator. Fig. 1(f) shows the eye diagrams for on-off keying (OOK) and four-state pulse amplitude modulation (PAM-4) signals at symbol rates of $64 \mathrm{GBd}$ and $100 \mathrm{GBd}$. At $64 \mathrm{GBd}$, the BER is below $10^{-9}$ for OOK and reaches $5 \times 10^{-5}$ for PAM-4. At $100 \mathrm{GBd}$ OOK, a BER of $4 \times 10^{-6}$ is measured, which is still well below the forward error correction (FEC) limit with $7 \%$ overhead. For 100 GBd PAM4 (200 Gbit/s line rate), we obtain a BER of $9 \times 10^{-3}$, which is just below the threshold of FEC with a $20 \%$ overhead. To the best of our knowledge, this represents the highest PAM-4 data rate so far demonstrated with modulators based on dielectric waveguides on the silicon platform.

\section{Summary}

We presented a capacitively coupled silicon-organic hybrid modulator with $3 \mathrm{~dB}$ EO bandwidth beyond $65 \mathrm{GHz}$ and a $U_{\pi} L$-product of $1.3 \mathrm{~V} \mathrm{~mm}$. We demonstrated $100 \mathrm{GBd}$ PAM-4 signaling with BER below the $20 \%$ FEC limit.

This work is supported by the European Research Council (ERC Consolidator Grant 'TeraSHAPE', \# 773248), by the DFG project PACE (\# KO 4192/9-1) within the Priority Programme "Electronic-Photonic Integrated Systems for Ultrafast Signal Processing" (SPP 2111), by the DFG project HIPES (\# KO 4192/6-1), by the Alfried Krupp von Bohlen und Halbach Foundation, by the Karlsruhe Nano Micro Facility (KNMF), and by the European Regional Development Fund (ERDF).

\section{References}

1. Azadeh, S. S. et al. Low V $\pi$ silicon photonics modulators with highly linear epitaxially grown phase shifters. Opt. Express 23, 23526 (2015).

2. Kieninger, C. et al. Ultra-high electro-optic activity demonstrated in a silicon-organic hybrid (SOH) modulator. Optica 5, 739-748 (2018).

3. Wolf, S. et al. Silicon-Organic Hybrid (SOH) Mach-Zehnder Modulators for $100 \mathrm{Gbit} / \mathrm{s}$ on-off Keying. Sci. Rep. 8, 1-13 (2018).

4. Ummethala, S. et al. THz-to-Optical Conversion in Wireless Communications Using an Ultra-Broadband Plasmonic Modulator. arXiv
Prepr. arXiv1812.04121 (2018).

5. Shi, S. \& Prather, D. W. Ultrabroadband electro-optic modulator based on hybrid silicon-polymer dual vertical slot waveguide. Adv. Optoelectron. 2011, (2011).

6. Konkol, M. R. et al. Dual slot modulator for millimeter wave photonics. Org. Photonic Mater. Devices XVI 8983, 898317 (2014).

7. Park, S. S. Properties of BaTiO3 films sputter deposited on PET for pulse power capacitors. Ferroelectrics 457, 97-104 (2013). 